\title{
3D Galactic extinction modelling
}

\author{
Richard J. Hanson and Coryn A. L. Bailer-Jones \\ Max-Planck-Institut für Astronomie, Königstuhl 17, 69117 Heidelberg, Germany \\ email: hanson@mia.de
}

\begin{abstract}
Using multi-band photometry we train a forward model to emulate the colour-change due to temperature and extinction for stars. We simultaneously solve for the astrophysical parameters (APs) extinction $A_{0}$, temperature $T_{\text {eff }}$ and distance modulus $\mu_{r}$ in a Bayesian framework for individual stars. We introduce an HRD prior to account for previous knowledge about the distribution of stars in the temperature- absolute magnitude plane (see also Bailer-Jones 2011). This allows us to infer distance information. We obtain the full three dimensional PDFs of all the stars in a field, allowing us to determine extinction-distance profiles for a given line of sight.
\end{abstract}

Keywords. methods: statistical - stars: statistics - ISM: dust, extinction

To build and train the forward model, we randomly select 4000 crossmatched stars with known temperatures from SEGUE/SDSS and latitudes above 70 degrees and photometry from SDSS DR8 (Aihara et al. 2011) and UKIDSS (Lawrence et al. 1999) LAS (Large Area Survey) DR9. We simulate and add extinction to these stars to build a grid in temperature and extinction for the eight resulting colours. We fit a thin-plate spline which models the smooth variation in parameter space well. Using a Gaussian noise model to compute the likelihood and a Hertzsprung-Russel diagram (HRD) prior to effectively use much of our knowledge of stellar evolution we compute the posterior distributions.

Further analysis reveals that including the NIR data from UKIDSS significantly improves the performance of the model as well as increasing the confidence of the inferred parameters. In practice we obtain systematic uncertainties of -0.08 mag and $-67 \mathrm{~K}$ for extinction and effective temperature, respectively. These are lower by a factor of roughly $4-5$ compared to using only SDSS photometry. Due to the degeneracy in these two parameters we obtain for the mean absolute error in the residuals $\operatorname{MAE}\left(A_{0}\right)=0.23 \mathrm{mag}$ and $\operatorname{MAE}\left(T_{\text {eff }}\right)=299 \mathrm{~K}$. Again, using only SDSS data, these values are $2-3$ times higher.

Not only does this method allow us to compute extinction, temperature and distance estimates for individual stars, we also obtain full three-dimensional PDFs to quantify our confidence in the results. These allow us to compute distance-extinction profiles for lines of sight, which take into account the probability and confidence for each parameter estimate and star, therefore resulting in statistically signifcant (weighted) means and uncertainties (error bars). Individual extinction estimates of stars, as well as in the limit of large distances, are generally consistent with maps of e.g. Schlegel, Finkbeiner \& Davis (1998). Slight differences due to the limitations of these methods are expected and seen.

For further information and plots, as well as the poster, please visit www.rhanson.de.

\section{References}

Aihara, H., et al. 2011, ApJS, 193, 29

Bailer-Jones, C. A. L. 2011, MNRAS, 411, 435

Lawrence A., et al. 2007, MNRAS, 379, 1599

Schlegel, D. J., Finkbeiner, D. P., \& Davis, M. 1998, ApJ, 500, 525 